\title{
Improved Stability Criteria of Generalized Recurrent Neural Networks with Time-varying Delays
}

\author{
Jinfang Zhang1 Yuanhua Qiao1 Jun Miao2 Lijuan Duan3 \\ ${ }^{1}$ College of Applied Science, Beijing University of Technology, Beijing, 100124, China \\ ${ }^{2}$ Institute of Computing Technology, Chinese Academy of Sciences, Beijing 100190, China \\ ${ }^{3}$ College of Computer Science, Beijing University of Technology, Beijing 100124, China
}

\begin{abstract}
In this paper, the problem on stability analysis of generalized recurrent neural networks with a time-varying delays is considered. Neither the differentiability, the monotony on these activation functions nor the differentiability on the time-varying delays are assumed. By employing a new Lyapunov-Krasovskii function, a linear matrix inequality (LMI) approach is developed to establish sufficient conditions for RNNs to be globally asymptotically stable. The proposed stability results are less conservative than some recently known ones in the literature. Finally an example is given to verify the effectiveness of the present criterion.

Index Terms: Recurrent neural networks; Global asymptotically stability; Time-varying delays; Linear matrix inequality
\end{abstract}

\section{Introduction}

It is well known that delayed neural networks have a wide range of applications in many fields such as pattern recognition, image processing, associative memory, and optimization problems [1-4]. Many interesting results on global asymptotic stability and global exponential stability have been obtained in recent years. It is worth mentioning that the obtained results in [5-12] are based on the following assumptions: $(i)$ the involved time-delays are constant delays [5-8] or with time-varying delays terms but continuously differentiable [9-12], and (ii) the activation functions are monotony or differentiability [13]. However, in many situations, time delays occur frequently and vary in an irregular fashion, and sometimes, they may be not continuously differentiable. In such case, those results can not be applied. The purpose of this works is to improve and complement the results in [5-13] and present a new criterion concerning, the global asymptotic stability of recurrent neural networks with time-varying delays, which is independent of the time-varying delays and does not require the differentiability of delay functions. An example is given to verify the effectiveness of the present criterion.

Notations. The notations are quite standard. Throughout this paper, $R^{n}$ and $R^{n \times m}$ denote, respectively, the $n$ dimensional Euclidean space and the set of all $n \times m$ real matrices. The superscript ' $T$ ' denotes matrix transposition. The notation $X \geq Y$ (respectively, $X>Y$ ) means that $X$ and $Y$ are symmetric matrices, and that $X-Y$ is positive semi-definite (respectively, positive definite). If $A$ is a matrix, $\lambda_{\max }(\cdot)$ (respectively, $\lambda_{\min }(\cdot)$ ) means the largest (respectively, smallest) eigenvalue of $A$. Sometimes, the arguments of a function or a matrix will be omitted in the analysis when no confusion arises.

\section{System description}

In this paper, we consider the following model:

$$
\dot{x}(t)=-C x(t)+A f(x(t))+B f(x(t-d(t)))+J \quad t \geq 0
$$

where $n$ corresponds to the number of units in a neural network $x(t)=\left(x_{1}(t), x_{2}(t), \ldots, x_{n}(t)\right)^{T} \in R^{n}$ is the neuron state vector; $f(x(t))=\left(f_{1}\left(x_{1}(t)\right), f_{2}\left(x_{2}(t)\right), \ldots, f_{n}\left(x_{n}(t)\right)\right)^{T}$,

$$
f(x(t-d(t)))=\left(f_{1}\left(x_{1}\left(t-d_{1}(t)\right)\right), f_{2}\left(x_{2}\left(t-d_{2}(t)\right), \ldots, f_{n}\left(x_{n}\left(t-d_{n}(t)\right)\right)\right)^{T} .\right.
$$

$f_{j}$ is activation function; $d_{j}(t)$ corresponds to the transmission delay and satisfies $0 \leq d_{j}(t) \leq d_{j} \quad\left(d_{j}\right.$ is a constant); $C=\operatorname{diag}\left(c_{1}, c_{2}, \ldots, c_{n}\right), c_{i}>0$ represents the rate constant with which the $i$ th unit will reset its potential to the resting state in isolation when it is disconnected from the networks and without external inputs; $A=\left(a_{i j}\right)_{n \times n}$ is referred to as the feedback matrix, $B=\left(b_{i j}\right)_{n \times n}$ represents the delayed feedback matrix. $J=\left(J_{1}, J_{2}, \ldots, J_{n}\right)^{T}$ is the constant external input vector. The initial condition of model (1) is of the form $x_{i}(s)=\phi_{i}(s), \quad-d \leq s \leq 0, \quad d=\max _{1 \leq i \leq n}\left\{d_{i}\right\}$, where $\phi_{i}(i=1,2, \ldots, n)$ is bounded and continuous on $[-d, 0]$.

Moreover, the neuron activation functions satisfy the following assumption:

(H)They are assumed to bounded and there exist two diagonal matrices $U=\operatorname{diag}\left(U_{1}, U_{2}, \ldots, U_{n}\right)$ and

$$
\begin{gathered}
F=\operatorname{diag}\left(F_{1}, F_{2}, \ldots, F_{n}\right) \text { such that } \\
U_{i} \leq \frac{f_{i}(\alpha)-f_{i}(\beta)}{\alpha-\beta} \leq F_{i}
\end{gathered}
$$

for all $\alpha, \beta \in R, \alpha \neq \beta, i=1,2, \ldots, n$.

To prove our main results, we need the following lemmas: Lemma 1 [14]. Given constant matrices $P, Q$ and $R$, where $P^{T}=P, Q^{T}=Q$, then the following LMI: 


$$
\left(\begin{array}{cc}
P & R \\
R^{T} & -Q
\end{array}\right)<0
$$

is equivalent to the following conditions

$Q>0, \quad P+R Q^{-1} R^{T}<0$.

Lemma 2 [15]. Supposed that $k_{1}, k_{2}, k_{3}: R_{+} \rightarrow R_{+}$are continuous and strict monotonous functions satisfying $k_{1}(0)=k_{2}(0)=0$, and when $s>0, k_{1}(s)>0, k_{2}(s)>0, k_{3}(s)>0$;

when $s \longrightarrow \infty, k_{1}(s) \rightarrow \infty$. then , the equilibrium point is globally asymptotically stable if there exist a continuous functions $V: R \times R^{n} \rightarrow R$, such that the inequality $k_{1}(\|x(t)\|) \leq V(t, x) \leq k_{2}\left(\|x\|_{d}\right), t \in R, x \in R^{n}$ and

$\dot{V}(t, x(t)) \leq-k_{3}(\|x(t)\|)$. here $\|x\|_{d}=\max _{t-d \leq \varsigma \leq t}\|x(\varsigma)\|$,

$\dot{V}$ is the time derivative of $V$ along the trajectories of (1).

\section{Main results and proofs}

Theorem 1. Let $x^{*}$ be the equilibrium point of the system (1).under assumption $(\mathrm{H})$, the equilibrium point is globally asymptotically stable if there exist a symmetric positive definite matric $P$, for diagonal matrices

$$
\begin{aligned}
& \Lambda=\operatorname{diag}\left(\lambda_{1}, \lambda_{2}, \ldots, \lambda_{n}\right)>0, \\
& \Delta=\operatorname{diag}\left(\delta_{1}, \delta_{2}, \ldots, \delta_{n}\right)>0, \\
& M_{1}=\operatorname{diag}\left(m_{11}, m_{12}, \ldots, m_{1 n}\right)>0 \text { and } \\
& M_{2}=\operatorname{diag}\left(m_{21}, m_{22}, \ldots, m_{2 n}\right)>0 \text { such }
\end{aligned}
$$

that the following LMI holds:

$$
\Omega=\left[\begin{array}{cc}
-P C-C P-2(F \Delta-U \Lambda) C-F M_{1} U & 0 \\
0 & -2 F M_{2} U \\
A^{T} \Gamma-C(\Delta-\Lambda)+M_{1}(F-U) & 0 \\
B^{T} \Gamma & (F-U) M_{2} \\
\Gamma A-C(\Lambda-\Delta)+M_{1}(F-U) & \Gamma B \\
0 & (F-U) M_{2} \\
2(\Lambda-\Delta) A-2 M_{1} & (\Lambda-\Delta) B \\
B^{T}(\Lambda-\Delta) & -2 M_{2}
\end{array}\right]<0
$$

Where $\Gamma=P+F \Delta-U \Lambda$ and all the matrices here are constant.

Proof. To simplify the asymptotical stability analysis of (1), we shift the equilibrium point $x^{*}$ of (1) to the origin by letting $y(t)=x(t)-x *$ and then the system (1) can be transformed to:

$$
\dot{y}(t)=-C y(t)+A g(y(t))+B g(y(t-d(t))), \quad t \geq 0
$$

where the transformed neuron activation function is:

$$
g(y(t))=f\left(y(t)+x^{*}\right)-f\left(x^{*}\right) .
$$

according to $(\mathrm{H})$,it can be easily checked that the transformed neuron activation function satisfy:

$$
U_{i} \leq \frac{g_{i}(\alpha)}{\alpha} \leq F_{i}, \quad i=1,2, \ldots, n .
$$

Now, we consider the following Lyapunov-Krasovskii functional candidate for model (4) as:

$$
\begin{aligned}
& V(y(t))= y^{T}(t) P y(t)+2 \sum_{i=1}^{n} \lambda_{i} \int_{0}^{y_{i}(t)}\left(g_{i}(s)-U_{i} s\right) d s \\
&\left.+2 \sum_{i=1}^{n} \delta_{i} \int_{0}^{y_{i}(t)}\left(F_{i} s-g_{i}(s)\right) d s\right), \quad t \geq 0 \\
& k_{1}(\|y(t)\|)=\lambda_{\min }(P)\|y(t)\|^{2} \leq V(y(t)) \\
& \leq \lambda_{\max }(P)\|y(t)\|^{2}+2(g(y(t))-U y(t))^{T} \Lambda y(t) \\
& \quad+2(F y(t)-g(y(t)))^{T} \Delta y(t) \\
& \leq \lambda_{\max }(P)\|y(t)\|^{2}+2 y^{T}(t)(F-U) \Lambda y(t) \\
& \quad+2 y^{T}(t)(F-U) \Delta y(t) \\
& \leq \lambda_{\max }(P)\|y(t)\|^{2}+2 y^{T}(t)(F-U)(\Lambda+\Delta) y(t) \\
&\left.\leq\left[\lambda_{\max }(P)+2 \lambda_{\max }((F-U)(\Lambda+\Delta))\right]\right]_{t-d \leq \varsigma \leq t} \max \|y(\varsigma)\|^{2} \\
&=k_{2}\left(\|y\|_{d}\right)
\end{aligned}
$$

Calculating the time derivative of $V(y(t))$ along the solution of system (4), we have

$$
\begin{aligned}
\dot{V}(y(t))= & 2 y^{T}(t) P[-C y(t)+A g(y(t)) \\
& +B g(y(t-d(t)))]+2 g^{T}(y(t))(\Lambda-\Delta) \\
{[-C y(t)} & +A g(y(t))+B g(y(t-d(t)))] \\
& +2 y^{T}(t)(F \Delta-U \Lambda)[-C y(t)+A g(y(t)) \\
+ & B g(y(t-d(t)))]
\end{aligned}
$$

On the other hand, in view of (2), we have

$$
\begin{aligned}
& (g(y(t))-F y(t))(g(y(t))-U y(t)) \leq 0 \\
& (g(y(t-d(t)))-F y(t-d(t))) \\
& (g(y(t-d(t)))-U y(t-d(t))) \leq 0
\end{aligned}
$$

Then consequently we have 


$$
\begin{aligned}
0 \leq & -2 \sum_{j=1}^{n} m_{1 j}\left(g_{j}\left(y_{j}(t)\right)-F_{j} y_{j}(t)\right)\left(g_{j}\left(y_{j}(t)\right)-U_{j} y_{j}(t)\right) \\
& -2 \sum_{j=1}^{n} m_{2 j}\left(g_{j}\left(y_{j}(t-d(t))\right)-F_{j} y_{j}(t-d(t))\right) \\
& \left(g_{j}\left(y_{j}(t-d(t))\right)-U_{j} y_{j}(t-d(t))\right) \\
= & -2(g(y(t))-F y(t))^{T} M_{1}(g(y(t))-U y(t)) \\
& -2\left(g(y(t-d(t))-F y(t-d(t)))^{T} M_{2}\right. \\
& g(y(t-d(t))-U y(t-d(t))))
\end{aligned}
$$

Denoting $\eta(t)=\left(y^{T}(t) y^{T}(t-d(t)) g^{T}(y(t)) g^{T}(y(t-d(t)))\right)^{T}$

and combing (8), (11), it is clearly that

$$
\dot{V}(y(t)) \leq \eta^{T}(t) \Omega \eta(t)
$$

where

$$
\begin{aligned}
& \Omega=\left[\begin{array}{cc}
-P C-C P-2(F \Delta-U \Lambda) C-F M_{1} U & 0 \\
0 & -2 F M_{2} U \\
A^{T} \Gamma-C(\Delta-\Lambda)+M_{1}(F-U) & 0 \\
B^{T} \Gamma & (F-U) M_{2}
\end{array}\right. \\
& \left.\begin{array}{cc}
\Gamma A-C(\Lambda-\Delta)+M_{1}(F-U) & \Gamma B \\
0 & (F-U) M_{2} \\
2(\Lambda-\Delta) A-2 M_{1} & (\Lambda-\Delta) B \\
B^{T}(\Lambda-\Delta) & -2 M_{2}
\end{array}\right]
\end{aligned}
$$

and $\Gamma=P+F \Delta-U \Lambda$.

Using the lemma 1, we obtain $\Omega<0$.

Following (3) and (12), we have

$$
\begin{aligned}
\dot{V}(y(t)) & \leq \lambda_{\max }(\Omega)\|y(t)\|^{2} \\
& =-\left(-\lambda_{\max }(\Omega)\right)\|y(t)\|^{2} \\
& =-k_{3}(\|y(t)\|) .
\end{aligned}
$$

With lemma 2, the equilibrium point $x^{*}$ of (1) is globally asymptotically stable. The proof of this theorem is now complete.

Remark 1.The assumption on the time-varying delays in this paper is bounded but not necessarily differentiable. However, the criterions in [7], [8] can only be applied to CNNs with constant delay, it is obvious that our results are more comprehensive and effective, in [12] the differentiability of the time-varying delays was required.

Remark 2. In $[5,8,12]$, the authors investigated the global asymptotic stability of equilibrium point of model (1) under the following assumptions (H1) and (H2) or (H3):

(H1)The activation functions $f_{i}(i=1,2, \ldots n)$

are bounded.
(H2)There exists a positive diagonal matrix $F$,

$$
F=\operatorname{diag}\left(F_{1}, F_{2}, \ldots F_{n}\right) \text { such that }
$$

$0 \leq \frac{f_{i}(\alpha)-f_{i}(\beta)}{\alpha-\beta} \leq F_{i}$ for all $\alpha, \beta \in R, \alpha \neq \beta$.

(H3)There exists a positive diagonal matrix $L=\operatorname{diag}\left(L_{1}, L_{2}, \ldots, L_{n}\right)$ such that

$\left|f_{i}(\alpha)-f_{i}(\beta)\right| \leq L|\alpha-\beta|$ for all $\alpha, \beta \in R$.

However, in this paper, the differentiability of activation functions is not required. In addition, the constants in assumption $(\mathrm{H})$ are allowed to be positive, negative or zero. Clearly, Assumption $(\mathrm{H})$ of this paper is weaker than those given in $[5,8,12]$.

Corollary1. Under assumption (H2), model (1) has a unique equilibrium point, which is globally asymptotic stable if there exist a positive definite matrix $P$ and four positive diagonal matrices $\Delta, \Lambda, M_{1}$ and $M_{2}$ such that the following LMIs hold:

$$
\left[\begin{array}{cccc}
-P C-C P-2 F \Delta C & 0 & \Gamma A-C(\Lambda-\Delta)+M_{1} F & \Gamma B \\
0 & 0 & 0 & F M_{2} \\
A^{T} \Gamma-C(\Delta-\Lambda)+M_{1} F & 0 & 2(\Lambda-\Delta) A-2 M_{1} & (\Lambda-\Delta) B \\
B^{T} \Gamma & F M_{2} & B^{T}(\Lambda-\Delta) & -2 M_{2}
\end{array}\right]<0
$$

where $\Gamma=P+F \Delta$.

Corollary2. Under assumption(H3), model(1)has a unique equilibrium point, which is globally asymptotic stable if there exist a positive definite matrix $P$ and four positive diagonal matrices $\Delta, \Lambda, M_{1}$ and $M_{2}$ such that the following LMIs hold:

$$
\begin{gathered}
{\left[\begin{array}{cc}
-P C-C P-2 F(\Delta+\Lambda) C+F^{2} M_{1} & 0 \\
0 & 2 F^{2} M_{2} \\
A^{T} \Gamma-C(\Delta-\Lambda)+2 M_{1} F & 0 \\
B^{T} \Gamma & 2 F M_{2} \\
\Gamma A-C(\Lambda-\Delta)+2 M_{1} F & \Gamma B \\
0 & 2 F M_{2} \\
2(\Lambda-\Delta) A-2 M_{1} & (\Lambda-\Delta) B \\
B^{T}(\Lambda-\Delta) & -2 M_{2}
\end{array}\right]<0}
\end{gathered}
$$

where $\Gamma=P+F(\Delta-\Lambda)$.

\section{Numerical example}

In this section, we present a simulation example so as to illustrate the usefulness of our main results. Our aim is to examine the global asymptotic stable of the delayed RNN (4) with network parameters given as follows:

$$
C=\left[\begin{array}{cc}
2.1 & 0 \\
0 & 2.3
\end{array}\right], A=\left[\begin{array}{cc}
-0.2 & 0.1 \\
-0.2 & 0.1
\end{array}\right],
$$




$$
\begin{gathered}
B=\left[\begin{array}{ll}
0.7 & 0.5 \\
0.5 & 0.4
\end{array}\right], J=\left[\begin{array}{l}
0 \\
0
\end{array}\right], \\
f_{1}(x)=0.5 \sin x, f_{2}(x)=\sin x .
\end{gathered}
$$

The activation functions satisfy Assumption $(\mathrm{H})$ with

$$
U=\left[\begin{array}{cc}
-0.5 & 0 \\
0 & -1
\end{array}\right], \quad F=\left[\begin{array}{cc}
0.5 & 0 \\
0 & 1
\end{array}\right]
$$

By the Matlab LMI Control Toolbox, we find a solution to the LMI in (3) as follows

$$
\begin{gathered}
P=\left[\begin{array}{cc}
0.0715 & -0.1010 \\
-0.1010 & 0.1427
\end{array}\right] \quad \Delta=\left[\begin{array}{cc}
0.3086 & 0 \\
0 & 0.3086
\end{array}\right] \\
\Lambda=\left[\begin{array}{cc}
0.3622 & 0 \\
0 & 0.3622
\end{array}\right] \\
M_{1}=\left[\begin{array}{cc}
0.3958 & 0 \\
0 & 0.3958
\end{array}\right] \quad M_{2}=\left[\begin{array}{cc}
0.2396 & 0 \\
0 & 0.2396
\end{array}\right]
\end{gathered}
$$

Therefore, it follows from Theorem 1 that the RNN (4) with given parameters is globally asymptotic stable.

\section{Conclusion}

This paper has provided new sufficient condition guaranteeing global asymptotic stability of the equilibrium point for the recurrent neural networks with time-varying delays and generalized activation functions. Neither the differentiability, he monotony on these activation functions nor the differentiability on the time-varying delays are required. The developed stability conditions are in terms of LMIs, which can be checked easily by recently developed algorithms that solves LMIs. Furthermore, the proposed stability conditions are less conservative than some recently known ones in the literature, which has been demonstrated via an example with simulation. For future investigation, we will apply other method to stochastic neural networks with Markovian jumping parameters and/or mixed time-delays, so that the main results can be extended.

\section{Acknowledgment}

This work was partially supported by National Natural Science Foundation of China (61070149, 60970087, 61272320, 61175115).

\section{References}

[1] Cichocki.A ,Unbehauen. R. Neural Networks for Optimization and Signal Processing, Wiley, Chichester, 1993.

[2] Cao. J.D. On stability of delayed cellular neural networks. Physics Letters A 1999; 261:303-308.

[3] Hopfield. J, Tank. D. Computing with neural circuits: a model. Science 1986; 233:625-633.

[4] Chua. L.O, Yang. L. Cellular neural networks: applications, IEEE Transactions on Circuits and Systems 35 (1988) 1273-1290.

[5] Ensari. T, Arik. S. New results for robust stability of dynamical neural networks with discrete time delays. Expert Systems with Applications 2010; 37:5925-5930.

[6] Li. X.D. Global exponential stability for a class of neural networks. Applied Mathematics Letters 2009;22:1235-1239.

[7] Singh. V. Novel global robust stability criterion for neural networks with delay. Chaos Solitons Fractals 2009; 1:348-353.

[8] Shen. Y.J, Yu. H, Jian. J.G. Delay-dependent global asymptotic stability for delayed cellular neural networks. Commun Nonlinear Sci and Numer Simulat 2009; 14:1057-1063.

[9] Li. X.D, Chen. Z. Stability properties for Hopfield neural networks with delays and impulsive perturbations. Nonlinear Analysis: Real World Applications 2009; 10:3253-3265.

[10] Xu. S.Y, Lam. J. A new approach to exponential stability analysis of neural networks with time-varying delays. Neural Networks 2006; 19:76-83.

[11] Shao. J.L, Huang. T.Z, Zhou. S. An analysis on global robust exponential stability of neural networks with time-varying delays. Neuro computing 2009; 72:1993-1998.

[12] Yu. W.W, Yao. L.L. Global robust stability of neural networks with time varying delays. Journal of Computational and Applied Mathematics 2007; 206:679-687.

[13] Liu. L.P, Han. Z.Z, Li. W.L. Global stability analysis of interval neural networks with discrete and distributed delays of neutral type. Expert Systems with Applications 2009; 36:7328-7331.

[14] Wang. Z.D. Liu. Y.Y. On global asymptotic stability of neural networks with discrete and distributed delays. Phys. Letts. 2005; A 345 (4-6):299-308.

[15] Hale. J.K, Lund. S.M. Introduction to the theory of functional differential equations. Applied Mathematical Sciences ,New York: springer,1991 